\title{
A Trajectory Generation Scheme for Precise and Safe Lunar Landing*
}

\author{
Ibrahim M MEHEDI** and Takashi KUBOTA*** \\ ** Department of Electrical Engineering and Information Systems \\ The University of Tokyo, 7-3-1 Hongo, Bunkyo-ku, Tokyo 113-8656, Japan \\ E-mail: mehedi@nnl.isas.jaxa.jp \\ *** Institute of Space and Astronautical Science \\ Japan Aerospace Exploration Agency, Sagamihara, Japan
}

\begin{abstract}
Execution of scientific objectives such as to investigate the central peaks of a big crater of the moon is thought to be the most scientifically interesting. Precise and safe autonomous landing ability is a productive issue for this investigation. Here it is proposed a scheme of trajectory generation for lunar descent. This research includes a sketch of a qualitative scheme on solutions of motion control equations for lunar descent vehicle from orbital speed condition down to the terminal descent situation. A trajectory generation algorithm is developed accumulating more than one step with variable thrust to mass ratio. Mathematical modeling, algorithm design, simulations and results are presented in this paper. In fact, the proposed trajectory generation scheme can readily be used to develop real-time guidance algorithm for future precise and safe lunar landing missions reducing the computational burden.
\end{abstract}

Key words : Precise Landing, Descent, Trajectory Generation, Algorithm Theory, Lunar Landing Mission

\section{Introduction}

Because the moon is widely regarded as the next step into space for us, different space agencies have begun new missions for lunar exploration. In fact lunar or planetary landing issues have received considerable attention since $1960 \mathrm{~s}^{(1)}$, but the future space exploration missions are required landing spacecraft on planets, moons, comets, and asteroids having different criteria for success ${ }^{(1)-(3),(5)-(9)}$. The similarity is that every mission entails some level of precise and safe landing capability especially for hazardous terrain to perform scientific region survey or sample return missions. After a long gap of Apollo program, the lunar maria are not attractive targets to explore. A landing site near the central peaks of a big crater is thought to be the most scientifically interesting. Such sites are completely unexplored by human and may give us clues about the origin of the moon. Surely it is a difficult and risky task to land close to the pre-selected landing zone with scientific value, such as near the central peaks of a crater, by an autonomous landing spacecraft on the moon because of the communication delay induced by the large distance ${ }^{(10)}$. Therefore, the traditional trajectory generation schemes and GNC modes using deep space network are not suitable for precise and safe lunar or planetary landing where all operations in the descent and landing phase must be done autonomously using on-board sensors and algorithms. It is also essential for a lunar lander to land vertically and softly on the surface ${ }^{(11)}$.

As illustrated in Figs. 1 and 2, the lunar guidance scheme takes the horizontally oriented spacecraft from orbital speed to almost vertical orientation and very low speed condition. The descent solution presented in this paper will have the spirit of gravity-turn descent solution $^{(2)-(5),(11)}$ nevertheless will develop the solution into a readily executable algorithm. This type of descent technique which entails that the lander thrust vector is oriented opposite to 


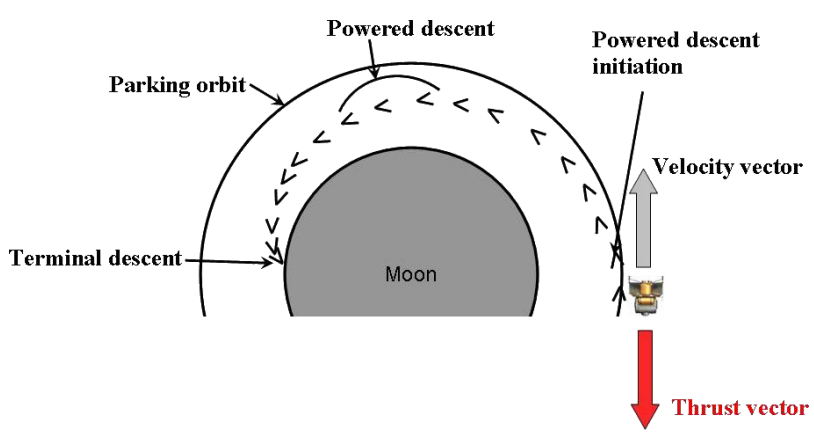

Fig. 1 Typical lunar landing trajectory

the velocity vector along the complete flight path of the vehicle. Using inertial measurement unit, the information about the velocity vector can be identified to insert as an input of attitude control system that can maintain thrust vector parallel to the velocity vector instantaneously but in opposite direction as shown in Fig.1. The great benefit of using gravity-turn descent is to have guaranteed upright landing, and fuel consumption is optimal.The design and implementation of a lunar descent is divided into two phases: the reference trajectory generation and the real time guidance. It is essential to avoid iterative, numerical optimization scheme and to reduce complexity for computing the reference trajectory. Further, a generic reference trajectory should be generated so that it can be implemented in a real time guidance algorithm unlike the reference to be in the form of two quartic polynomials ${ }^{(1)}$.

This paper introduces a qualitative solution of spacecraft motion equations that implements a complete depiction of the descent module motion from orbital speed conditions down to the final landing state on a homogeneous spherical lunar surface. Centrifugal forces are retained during descent process. These followed by developing an analytical reference trajectory generation algorithm that will be used in real time to promptly and consistently generate two dimensional trajectories ahead of descent initiation or re-target to another landing site after descent initiation.

This paper is structured as follows. Section 2 describes the fundamentals of spacecraft motion equations for lunar descent. Advanced analytical solutions to the equations for speed, time, vertical range, and horizontal distance as a function of velocity vector pitch angle are presented in Section 3. Designing a reference trajectory algorithm and simulated responses are demonstrated in detail in Section 4. Finally Section 5 contains the conclusion.

\section{Dynamics of lunar lander}

In order to simplify the development of the lunar guidance scheme, the equations of motion can be developed in a maneuver reference frame defined relative to the lunar surface. Figure 3 shows description of maneuver reference frame demonstrating the states and forces with respect to the LVLH reference frame denoted by $L$. It also shows the relationship of the maneuver frame denoted by $M$ to the LVLH unit vectors. Fundamental three dimensional equations of motion to describe the spacecraft proposition concerning a uniform sphere-shaped lunar body ${ }^{(2)}$ are divided into two parts. One is the equations of spacecraft motion for dynamic states as follow:

$$
\begin{aligned}
& \dot{u}(t)=g_{l} \cos \alpha-N \cos \beta \\
& \dot{\alpha}(t)=\frac{1}{u}\left[\left(\frac{u^{2}}{y+y_{l}}-g_{l}\right) \sin \alpha-N \sin \beta \cos \varphi\right] \\
& \dot{\psi}(t)=\frac{1}{u \sin \alpha}[N \sin \beta \sin \varphi]
\end{aligned}
$$

where $u$ is spacecraft velocity vector magnitude or spacecraft speed, $g_{l}$ is lunar gravitational acceleration, $N$ is ratio of thrust $F$ and vehicle mass $m, \alpha$ is the pitch angle of the vehicle 


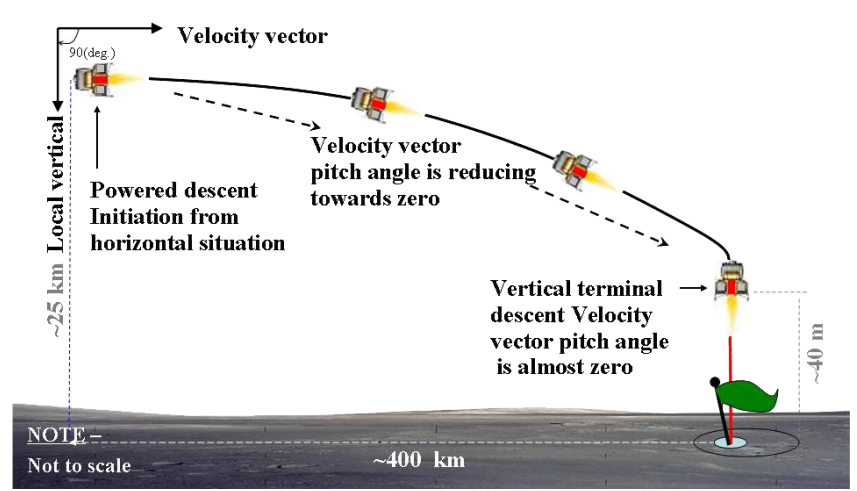

Fig. 2 Reference descent trajectory to land on Moon. The lunar descent guidance algorithm takes the horizontally oriented spacecraft from orbital speeds at a point hundreds of kilometers from the desired landing point to the landing point at an almost vertical orientation and very low speed

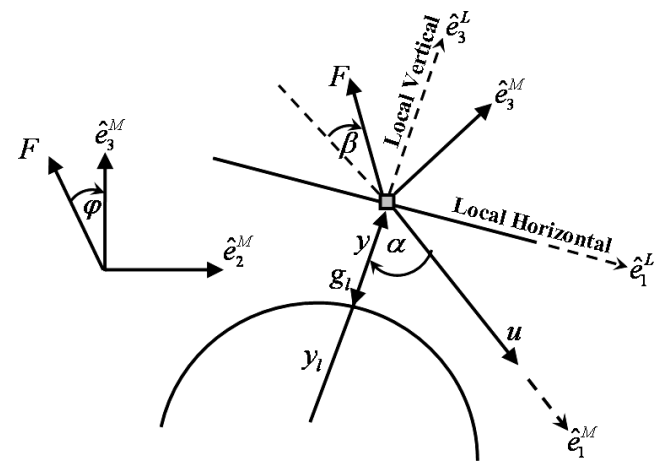

Fig. 3 Schematic diagram of lunar descent reference frame

velocity vector relative to the local vertical, $\beta$ is angle of thrust vector relative to reverse direction of spacecraft velocity, $y$ is altitude of the spacecraft from lunar surface, $y_{l}$ is lunar radius, $\psi$ is cross range angle, and $\varphi$ is thrust roll angle.

The remaining part to describe the fundamental equations of motion for kinematics states are

$$
\begin{aligned}
& \dot{y}(t)=-u \cos \alpha \\
& \dot{x}(t)=u \sin \alpha \cos \psi \frac{y_{l}}{y+y_{l}} \\
& \dot{c}(t)=u \sin \alpha \sin \psi \frac{y_{l}}{y+y_{l}}
\end{aligned}
$$

where $x$ and $c$ are horizontal span and cross range distance respectively. To facilitate the simplification of mathematical operation, roll control states are held at zero $(\varphi(t)=0)$ and $(\dot{\varphi}(t)=0)$. To activate the system as a plane motion, the initial states are initialized to zero $(c(0)=0),(\psi(0)=0)$ and $(\dot{\psi}(0)=0)$. Consequently the above governing equations are reduced to their two-dimensional form where equations (1) and (4) remain same. Required changes are observed as follow:

$$
\dot{\alpha}(t)=\frac{1}{u}\left[\left(\frac{u^{2}}{y+y_{l}}-g_{l}\right) \sin \alpha-N \sin \beta\right]
$$

and $\dot{x}(t)=u \sin \alpha \frac{y_{l}}{y+y_{l}}$. It is reasonable to assume that $y<<y_{l}$ in order that $\frac{y_{l}}{y+y_{l}} \approx 1$. Then the equation for horizontal span becomes

$$
\dot{x}(t)=u \sin \alpha
$$




\section{Advanced Analytical solution for differential equation}

To solve those governing equations qualitatively, it is essential that the right hand sides of the equations are kept as a function of velocity vector pitch angle $\alpha$ considering an important assumption for centrifugal acceleration term ${ }^{(14)}$. In contrast, the conventional solutions have ignored this postulation.Using initial values for mass and gravity is an uncomplicated assumption for qualitative solution for generating reference descent trajectory. For centrifugal acceleration term, however a constant value $\Gamma$ can be logically chosen which is defined as the ratio between centrifugal acceleration and lunar gravitational acceleration such that $\Gamma=\frac{\frac{u^{2}}{y+y_{l}}}{g_{l}}$. Therefore, $\frac{u^{2}}{y+y_{l}}-g_{l}=-(1-\Gamma) g_{l}$. With these assumptions and making consistent with the traditional gravity-turn descent work ${ }^{(2),(4),(5)}$, speed can be recognized by following differential equation formulating as a function of velocity vector pitch angle $\alpha$.

$$
\dot{u}(\alpha)=\dot{u}(t) / \dot{\alpha}(t)=u \frac{g_{l} \cos \alpha-N}{-(1-\Gamma) g_{l} \sin \alpha}
$$

or,

$$
\frac{d u}{u}=\left[\frac{g_{l} \cos \alpha-N}{-(1-\Gamma) g_{l} \sin \alpha}\right] d \alpha
$$

This equation can now be directly integrated to obtain the descent velocity $u$ as a function of the velocity vector pitch angle $\alpha$ as

$$
u(\alpha)=u_{0} e^{\int_{\alpha_{0}}^{\alpha}\left[\frac{g_{l} \cos \alpha-N}{-(1-\Gamma) g_{l} \sin \alpha}\right] d \alpha}
$$

Now

$$
\int_{\alpha_{0}}^{\alpha}\left[\frac{g_{l} \cos \alpha-N}{-(1-\Gamma) g_{l} \sin \alpha}\right] d \alpha=\ln \left[\frac{\sin \alpha}{\sin \alpha_{0}}\right]^{\frac{-1}{(1-\Gamma)}}+\ln \left[\frac{\tan \frac{\alpha_{0}}{2}}{\tan \frac{\alpha}{2}}\right]^{\frac{-N}{(1-\Gamma) g_{l}}}
$$

therefore,

$$
u(\alpha)=u_{0}\left[\frac{\sin \alpha}{\sin \alpha_{0}}\right]^{\frac{-1}{(1-\Gamma)}}\left[\frac{\tan \frac{\alpha_{0}}{2}}{\tan \frac{\alpha}{2}}\right]^{\frac{-N}{(1-\Gamma) g_{l}}}
$$

as $\tan \left(\frac{\alpha}{2}\right)=\frac{1-\cos \alpha}{\sin \alpha}$ and let that $\tau=\frac{1}{1-\Gamma} \& \rho=\frac{N}{g_{l}}$. Where $\rho>0$ in order that

$$
u(\alpha)=u_{0}\left[\frac{\sin \alpha}{\sin \alpha_{0}}\right]^{-\tau}\left[\frac{\frac{1-\cos \alpha_{0}}{\sin \alpha_{0}}}{\frac{1-\cos \alpha}{\sin \alpha}}\right]^{-\tau \rho}
$$

where $\tau=\frac{1}{1-\Gamma}$ is a measure of the centrifugal acceleration term. Then the solution for speed currently obtains the shape

$$
u(\alpha)=u_{0}\left(\frac{\sin \alpha}{\sin \alpha_{0}}\right)^{-\tau(1+\rho)}\left(\frac{1-\cos \alpha_{0}}{1-\cos \alpha}\right)^{-\tau \rho}
$$

Now the descent time $t_{D}$, vertical range $y(\alpha)$ and horizontal span $x(\alpha)$ are resolved in an identical manner of the conventional gravity turn descent solution as follow

$$
\begin{aligned}
& \dot{t}_{D}=\frac{1}{\dot{\alpha}(t)}=\frac{-\tau u(\alpha)}{g_{l} \sin \alpha}=\frac{-\tau u_{0}}{g_{l}} \frac{\left(1-\cos \alpha_{0}\right)^{-\tau \rho}}{\left(\sin \alpha_{0}\right)^{-\tau(1+\rho)}} \frac{(\sin \alpha)^{-\tau(1+\rho)-1}}{(1-\cos \alpha)^{-\tau \rho}} \\
& \dot{t}_{D}=G_{t_{D}} \frac{(\sin \alpha)^{-\tau(1+\rho)-1}}{(1-\cos \alpha)^{-\tau \rho}}
\end{aligned}
$$

where

$$
G_{t_{D}}=\frac{-\tau u_{0}}{g_{l}} \frac{\left(1-\cos \alpha_{0}\right)^{-\tau \rho}}{\left(\sin \alpha_{0}\right)^{-\tau(1+\rho)}}
$$

Now for vertical range, 


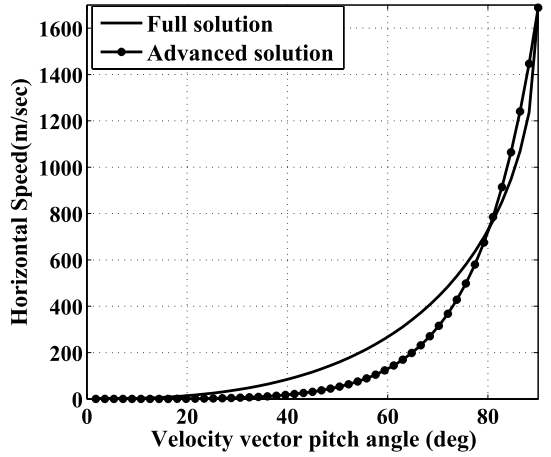

(a)

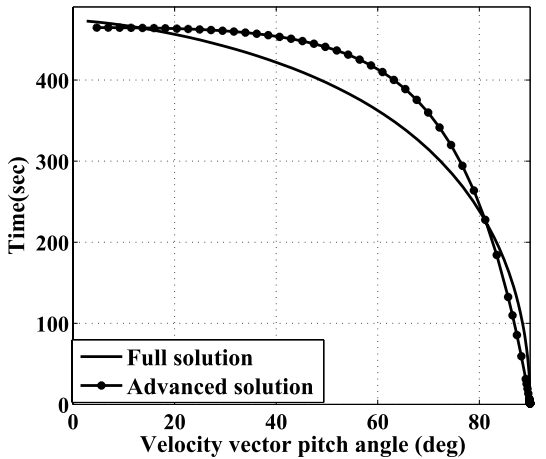

(c)

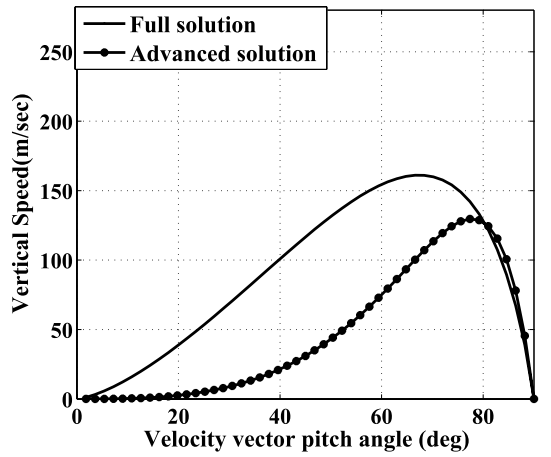

(b)

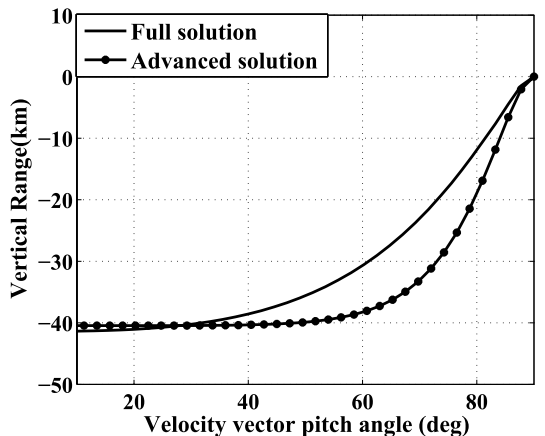

(d)

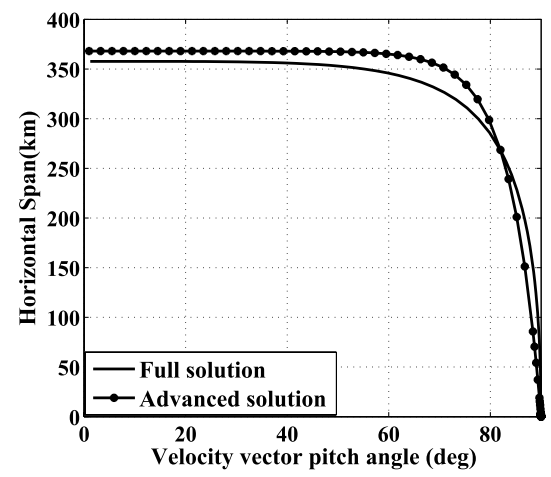

(e)

Fig. 4 An assessment for analytical solutions and fully integrated solutions of lunar descent speed, time, vertical range, and horizontal span given $\tau=2$ 


$$
\begin{aligned}
& \dot{y}(\alpha)=\frac{\dot{y}(t)}{\dot{\alpha}(t)}=\frac{\tau u_{0}^{2}}{g_{l}} \frac{\left(1-\cos \alpha_{0}\right)^{-2 \tau \rho}}{\left(\sin \alpha_{0}\right)^{-2 \tau(1+\rho)}} \frac{(\sin \alpha)^{-2 \tau(1+\rho)} \cos \alpha}{(1-\cos \alpha)^{-2 \tau \rho} \sin \alpha} \\
& \dot{y}(\alpha)=G_{y} \frac{(\sin \alpha)^{-2 \tau(1+\rho)-1} \cos \alpha}{(1-\cos \alpha)^{-2 \tau \rho}} s
\end{aligned}
$$

where

$$
G_{y}=\frac{\tau u_{0}^{2}}{g_{l}} \frac{\left(1-\cos \alpha_{0}\right)^{-2 \tau \rho}}{\left(\sin \alpha_{0}\right)^{-2 \tau(1+\rho)}}
$$

and for horizontal span,

$$
\begin{aligned}
& \dot{x}(\alpha)=\frac{\dot{x}(t)}{\dot{\alpha}(t)}=\frac{-\tau u_{0}^{2}}{g_{l}} \frac{\left(1-\cos \alpha_{0}\right)^{-2 \tau \rho}}{\left(\sin \alpha_{0}\right)^{-2 \tau(1+\rho)}} \frac{(\sin \alpha)^{-2 \tau(1+\rho)}}{(1-\cos \alpha)^{-2 \tau \rho}} \\
& \dot{x}(\alpha)=G_{x} \frac{(\sin \alpha)^{-2 \tau(1+\rho)}}{(1-\cos \alpha)^{-2 \tau \rho}}
\end{aligned}
$$

where

$$
G_{x}=\frac{-\tau u_{0}^{2}}{g_{l}} \frac{\left(1-\cos \alpha_{0}\right)^{-2 \tau \rho}}{\left(\sin \alpha_{0}\right)^{-2 \tau(1+\rho)}}
$$

It is evoked that the $\tau$ is the measure of the relation between centrifugal acceleration and lunar gravitational acceleration terms. With the purpose of integration for above equations in a qualitative manner the value for $\tau$ must be an integer. This entails $\tau=1,2,3,4 \ldots \ldots$. Instead of this solution, directly the ratio $\Gamma$, which is mentioned earlier, can be chosen some fractional values to make $\tau$ an integer. But the authors found better results having directly the integer logical values to get a qualitative integration of these equations. Choosing a logical values directly for the $\tau$ proves more preciseness in approximation as well. The influences of differing the constant $\tau$ is demonstrated in previous work ${ }^{(14)}$. Unlike values $(1,2,3,4,5, \ldots)$ for $\tau$ are employed into equations (15), (17), (20) and (23) and these equations are numerically integrated with constant approximate values for $g_{l}$ and $N$ whereas $g_{l}=1.623 \mathrm{~m} / \mathrm{sec}^{2}$ and $N$ $=5 \mathrm{~N} / \mathrm{kg}$. Initial and final values for the velocity vector pitch angle $\alpha$ is taken $90^{\circ}$ and $0^{0}$ while the initial speed $u_{0}$ is considered as approximate orbital speed, $1688 \mathrm{~m} / \mathrm{sec}$. In contrast of this advanced solution, full numerical integrated resolution to equations (1), (2), (4) and (5), and traditional gravity-turn solutions to equations ${ }^{(2),(4),(5)}$ are performed for comparison taking same approximation for $\beta, g_{l}, N, \alpha$ and $u_{0}$ as it is made into equations (15), (17), (20) and (23) while no estimation are made about the centrifugal acceleration.

A comprehensive evaluation of this advanced solution with traditional gravity-turn solution, and a numerically integrated solution to the full equations of lunar module descent are exposed in this investigation. It can be noted that varying $\tau$ has reasonable impact on different responses for speed, time, vertical range and horizontal span for lunar descent scheme. The largest impact is observed on the final vertical range variation. The centrifugal acceleration effectively adjusts the rate of change of the vehicle velocity vector pitch angle which impacts the direction of the velocity vector. Therefore, the term $\tau$ directly influences the vertical range of the trajectory. From the assessment of the various values for $\tau$, a value of $\tau=2$ emerges to be a realistic number and improves on different responses of advanced solutions for speed, time, vertical range and horizontal span over traditional solutions.

Now, from the latest shape of differential equations with $\tau=2$ the following results are obtained through mathematical derivations. For time

$$
t_{D}(\alpha)=G_{t_{D}}\left[\frac{1}{4 \rho\left(\rho^{2}-1\right)}(1-\cos \alpha)^{2 \rho}\left(2 \rho^{2}(\csc \alpha)^{2}+2 \rho \cot \alpha \csc \alpha-1\right)(\sin \alpha)^{-2 \rho}\right]+C_{t}
$$

let

$$
T(\alpha)=G_{t_{D}}\left[\frac{1}{4 \rho\left(\rho^{2}-1\right)}(1-\cos \alpha)^{2 \rho}\left(2 \rho^{2}(\csc \alpha)^{2}+2 \rho \cot \alpha \csc \alpha-1\right)(\sin \alpha)^{-2 \rho}\right](26)
$$




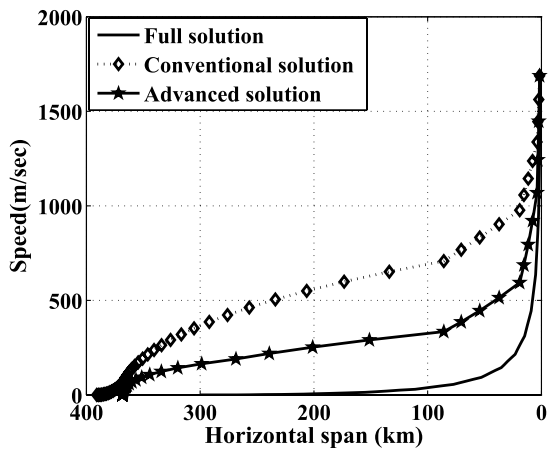

(a)

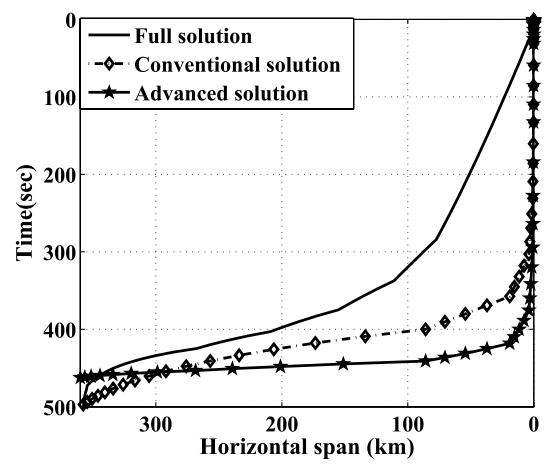

(b)

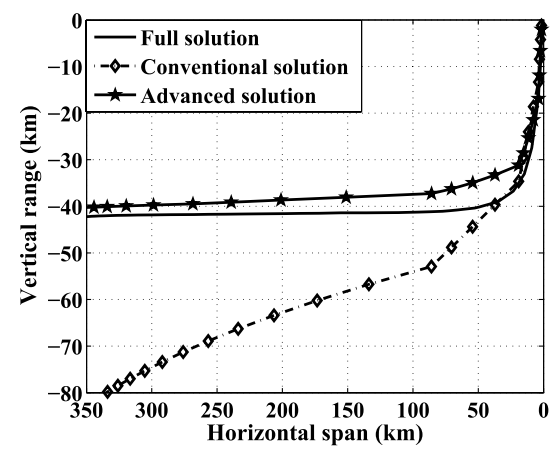

(c)

Fig. 5 An assessment for analytical solutions, conventional solution, and fully integrated solutions of lunar descent time, vertical range, and speed as a function of horizontal span

and

$$
C_{t}=t_{0}-T\left(\alpha_{0}\right)
$$

where $t_{0}$ is the initial time and at the time of descent initiation, $\alpha=\alpha_{0}$. Such that,

$$
t_{D}\left(\alpha_{0}\right)=T\left(\alpha_{0}\right)+C_{t}=t_{0}
$$

Now for vertical range

$$
y(\alpha)=G_{y} \frac{(\sin \alpha)^{-4 \rho-4}}{(1-\cos \alpha)^{-4 \rho}}\left[\frac{\left(32 \rho^{2}-8\right)+2 \rho\left(16 \rho^{2}+3 \cos 2 \alpha-7\right) \cos \alpha}{32\left(4 \rho^{4}-5 \rho^{2}+1\right)}-\frac{24 \rho^{2}(\sin \alpha)^{2}+3(\sin \alpha)^{4}}{32\left(4 \rho^{4}-5 \rho^{2}+1\right)}\right]+C_{y}
$$

let

$$
V(\alpha)=G_{y} \frac{(\sin \alpha)^{-4 \rho-4}}{(1-\cos \alpha)^{-4 \rho}}\left[\frac{\left(32 \rho^{2}-8\right)+2 \rho\left(16 \rho^{2}+3 \cos 2 \alpha-7\right) \cos \alpha}{32\left(4 \rho^{4}-5 \rho^{2}+1\right)}-\frac{24 \rho^{2}(\sin \alpha)^{2}+3(\sin \alpha)^{4}}{32\left(4 \rho^{4}-5 \rho^{2}+1\right)}\right]
$$

and

$$
C_{y}=y_{0}-V\left(\alpha_{0}\right)
$$

where $y_{0}$ is initial altitude and at the time of descent initiation, $\alpha=\alpha_{0}$. Such that,

$$
y\left(\alpha_{0}\right)=V\left(\alpha_{0}\right)+C_{y}=y_{0}
$$

Finally for for horizontal span

$$
x(\alpha)=G_{x} \frac{(\sin \alpha)^{-4 \rho-4} \sin \alpha}{(1-\cos \alpha)^{-4 \rho}}\left[\frac{128 \rho^{3}+24 \rho \cos 2 \alpha-32 \rho}{2\left(256 \rho^{4}-160 \rho^{2}+9\right)}+\frac{\left(96 \rho^{2}-9\right) \cos \alpha+3 \cos 3 \alpha}{2\left(256 \rho^{4}-160 \rho^{2}+9\right)}\right]+C_{x}
$$


let

$$
H(\alpha)=G_{x} \frac{(\sin \alpha)^{-4 \rho-4} \sin \alpha}{(1-\cos \alpha)^{-4 \rho}}\left[\frac{128 \rho^{3}+24 \rho \cos 2 \alpha-32 \rho}{2\left(256 \rho^{4}-160 \rho^{2}+9\right)}+\frac{\left(96 \rho^{2}-9\right) \cos \alpha+3 \cos 3 \alpha}{2\left(256 \rho^{4}-160 \rho^{2}+9\right)}\right]
$$

and

$$
C_{x}=x_{0}-H\left(\alpha_{0}\right)
$$

where $x_{0}$ is initial value of horizontal span and at the time of descent initiation, $\alpha=\alpha_{0}$. Such that,

$$
x\left(\alpha_{0}\right)=H\left(\alpha_{0}\right)+C_{x}=x_{0}
$$

A comparison is shown in Fig. 4. for the advanced analytical solutions of speed, time, vertical range, and horizontal span given $\tau=2$ to the fully integrated solutions to equations (1), (2), (4) and (5) where $\beta=0$. The fully integrated solution assumes a constant lunar gravitational acceleration, $g_{l}$, and constant thrust to mass ratio, $N$, but does not guess a constant centrifugal acceleration. These figures demonstrate the trajectory discrepancy that the guidance will be required to remove.

It is often useful to investigate the lunar descent trajectory responses as a function of horizontal span. Figure 5 shows different parameters like vehicle speed, necessary time, and vertical range of descent in terms of horizontal span. In this figure a comparison is demonstrated for different trajectory responses of spacecraft descent on lunar surface while the governing equations are solved by complete integration method, conventional illumination ${ }^{(14)}$, and the advanced analytical scheme. All these results are evaluated using the same initial and final valued parameters applied previously.

Table 1 Elapse time comparison for Computing (second)

\begin{tabular}{cccc} 
No. & Full Solution & Conv. Solution & Adv. Analytical Solution \\
\hline 01 & 3.7188 & 0.0313 & 0.0156 \\
02 & 3.6406 & 0.0313 & 0.0313 \\
03 & 3.7500 & 0.0313 & 0.0156 \\
04 & 3.8125 & 0.0313 & 0.0313 \\
05 & 3.7031 & 0.0313 & 0.0156 \\
06 & 3.6406 & 0.0313 & 0.0313 \\
07 & 3.6406 & 0.0313 & 0.0313 \\
08 & 4.5938 & 0.0234 & 0.0156 \\
09 & 6.0938 & 0.0312 & 0.0496 \\
10 & 5.2969 & 0.0250 & 0.0313 \\
\hline AVE. & 4.1891 & 0.0299 & 0.0269 \\
\hline
\end{tabular}

While the on line trajectory generation is a great challenge for lunar or planetary landing, it becomes useful to compare elapsed time spent to solve the numerical calculations producing trajectory response on board. Table 1 shows 10 number of different observations of computing time performance analysis for different schemes using desktop computer. Here it can be mentioned that the speed of computer used for this experiment is $2.66 \mathrm{GHz}$, which is 13.33 times higher than the current available on-board computer with only $200 \mathrm{MHz}$ speed. Among different responses of proposed advanced solutions, response for taking $\tau=2$ is specially observed in this study because this response is much attractive than the traditional scheme. Although the required computing time is similar between conventional scheme and the proposed advanced scheme, but significantly less required executing time is observed in terms of full solution. According to this elapsed time analysis both conventional and proposed advanced schemes are quicker than the complete numerical solution. But it is already established that the proposed advanced method of solution is more advantageous than the conventional solutions ${ }^{(14)}$.

\section{Designing a reference trajectory generation algorithm}

With the help of previously derived equations, a reference trajectory generation algorithm can be designed which will be a function of horizontal span, vertical range, speed, and velocity 
vector pitch angle. This type of trajectory is unspecified to any certain location on the lunar surface and it is a great benefit of this type of reference trajectory generation technique. From equation (29) and (33) it can be assumed that

$$
\begin{aligned}
& Y(\alpha, \rho)=\frac{\left(32 \rho^{2}-8\right)+2 \rho\left(16 \rho^{2}+3 \cos 2 \alpha-7\right) \cos \alpha}{32\left(4 \rho^{4}-5 \rho^{2}+1\right)}-\frac{24 \rho^{2}(\sin \alpha)^{2}+3(\sin \alpha)^{4}}{32\left(4 \rho^{4}-5 \rho^{2}+1\right)}(37) \\
& X(\alpha, \rho)=-\sin \alpha\left[\frac{128 \rho^{3}+24 \rho \cos 2 \alpha-32 \rho}{2\left(256 \rho^{4}-160 \rho^{2}+9\right)}+\frac{\left(96 \rho^{2}-9\right) \cos \alpha+3 \cos 3 \alpha}{2\left(256 \rho^{4}-160 \rho^{2}+9\right)}\right](38)
\end{aligned}
$$

and

$$
\frac{2 u(\alpha)^{2}}{g_{l}}=\frac{2 u_{0}^{2}}{g_{l}} \frac{\left(1-\cos \alpha_{0}\right)^{-4 \rho}}{\left(\sin \alpha_{0}\right)^{-4(1+\rho)}} \frac{(\sin \alpha)^{-4 \rho-4}}{(1-\cos \alpha)^{-4 \rho}}
$$

Therefore,

$$
y(\alpha)=y_{0}+\frac{2 u(\alpha)^{2}}{g_{l}} Y(\alpha, \rho)-\frac{2 u_{0}^{2}}{g_{l}} Y\left(\alpha_{0}, \rho\right)
$$

and

$$
x(\alpha)=x_{0}+\frac{2 u(\alpha)^{2}}{g_{l}} X(\alpha, \rho)-\frac{2 u_{0}^{2}}{g_{l}} X\left(\alpha_{0}, \rho\right)
$$

where the equation for speed obtains the shape

$$
u(\alpha)=u_{0}\left[\frac{\sin \alpha}{\sin \alpha_{0}}\right]^{-2(1+\rho)}\left[\frac{1-\cos \alpha_{0}}{1-\cos \alpha}\right]^{-2 \rho}
$$

To develop an algorithm for generating reference trajectory it is comprised of $n$ steps where thrust to mass ratio, $N$ is unchanged over each step and the combination of all the steps results in a collective vertical range and horizontal span. Different steps are joined by the continuity conditions that the speed and velocity vector pitch angle be continuous over each link. Let the initial vertical range and horizontal span of the complete trajectory are $y_{0}$ and $x_{0}$ and terminal values of these parameters are $y_{t}$ and $x_{t}$ as shown in Fig. 6. Now the complete displacements in vertical and horizontal direction are characterized by

$$
\delta y=-y_{0}+y_{t}=\sum_{i=1}^{n} \delta y_{i}
$$

and

$$
\delta x=x_{t}-x_{0}=\sum_{i=1}^{n} \delta x_{i}
$$

where $\delta y$ and $\delta x$ are complete paths in vertical and horizontal directions that can be traveled by the landing vehicle during powered descent phase. In equations (43) and (44), $\delta y$ and $\delta x$ define the total path starting from orbital condition and ending at the terminal point. The terminal speed and the terminal velocity vector pitch angle for the total trajectory are also associated with all the steps by the fact that final velocity vector pitch angle, $\alpha_{t}$, is the same as the $n^{\text {th }}$ vector pitch angle, $\alpha_{n}$ and the terminal speed, $u_{t}$ is the same as the $n^{\text {th }}$ speed, $u_{n}$. From equations (40) and (41) the individual step's change in vertical range and horizontal span are shown by

$$
\begin{aligned}
& \delta y_{i}=\frac{2 u_{i}^{2}}{g_{l}} Y\left(\alpha_{i}, \rho_{i}\right)-\frac{2 u_{i-1}^{2}}{g_{l}} Y\left(\alpha_{i-1}, \rho_{i}\right) \\
& \delta x_{i}=\frac{2 u_{i}^{2}}{g_{l}} X\left(\alpha_{i}, \rho_{i}\right)-\frac{2 u_{i-1}^{2}}{g_{l}} X\left(\alpha_{i-1}, \rho_{i}\right)
\end{aligned}
$$

where $\alpha_{i-1}$ and $\alpha_{i}$ are the velocity vector pitch angle at the beginning and at the end of $i^{\text {th }}$ step. Moreover, the speed at the end of $i^{\text {th }}$ step is known by 


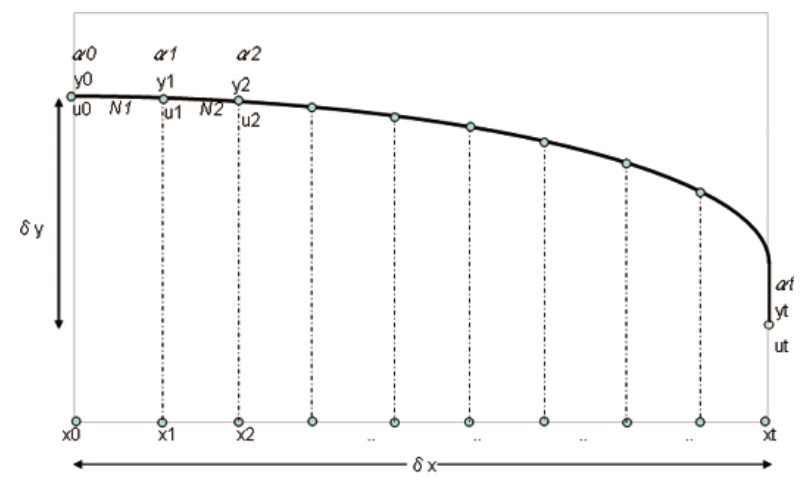

Fig. $6 n$ step reference trajectory

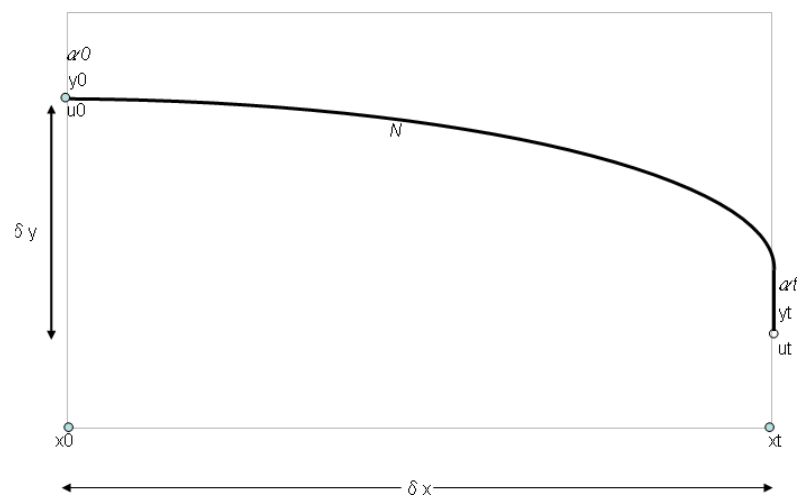

Fig. 7 Single step reference trajectory

$$
u_{i}=u_{i-1}\left[\frac{\sin \alpha_{i}}{\sin \alpha_{i-1}}\right]^{-2\left(1+\rho_{i}\right)}\left[\frac{1-\cos \alpha_{i-1}}{1-\cos \alpha_{i}}\right]^{-2 \rho_{i}}
$$

Here $u_{i-1}$ and $u_{i}$ are the speed at the beginning and at the end of $i^{t h}$ step and $\rho_{i}=\frac{N_{i}}{g_{l}}$ in which $N_{i}=\frac{F}{m}$ and $\rho_{i}$ are constant over all the steps. Actually the objective is stipulate the traveling distance both in vertical and horizontal direction by evaluating the velocity vector pitch angle and speed at the beginning and end of the total trajectory $\left(\alpha_{0}, \alpha_{t}, u_{0}, u_{t}\right)$. This would result in a preferred total vertical range and horizontal span given a set of values for $n$ values of $N_{i}$.

\subsection{Single step trajectory}

If a single step is considered as shown in Fig. 7 to describe the total trajectory, there exist only three equations (equations (43) and (44) where $n=1$ and equation (47) where $i=1$ ) with seven variables $\left(\alpha_{0}, \alpha_{1}, u_{0}, u_{1}, \delta y, \delta x, N\right)$, given the wish to identify six of those parameters $\left(\alpha_{0}, \alpha_{1}, u_{0}, u_{1}, \delta y, \delta x\right)$. This single step reference trajectory generation algorithm is rapidly over-constrained and this is the major difficulty. This problem can be overcome to bring under constraints by accumulating more steps to the solution.

\subsection{Double step trajectory}

If two steps are considered with the same objective (identify $\alpha_{0}, \alpha_{t}, u_{0}, u_{t}, \delta y, \delta x$ ) as stated before, the problems become constrained. There exist two equations (43) and (44), where $n=$ 2 and equation (47) where $i=1$ and $i=2$ with the variables $\left(\alpha_{0}, \alpha_{1}, \alpha_{2}, u_{0}, u_{1}, u_{2}, \delta y, \delta x, N_{1}, N_{2}\right)$. The task is to figure out $\alpha_{1}, u_{1}, N_{1}$ and $N_{2}$ given the other six predefined variables $\left(\alpha_{0}, \alpha_{2}, u_{0}, u_{2}\right.$, $\delta y, \delta x)$. Actually here, $\alpha_{2}$ and $u_{2}$ are the same as terminal values for velocity vector pitch angle and speed respectively. Figure 8 shows the schematic of flight path for double steps algorithm for reference trajectory.

It is easy to compute $u_{1}$ if $\alpha_{1}, N_{1}$ and $N_{2}$ are already known together with $\alpha_{0}$ and $u_{0}$ or 


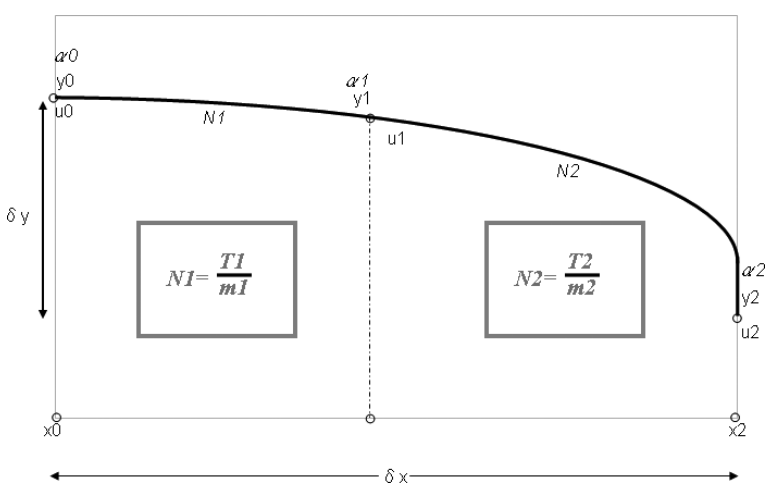

Fig. 8 Double steps reference trajectory

$\alpha_{2}$ and $u_{2}$ :

$$
\begin{aligned}
& u_{1}=u_{0}\left[\frac{\sin \alpha_{1}}{\sin \alpha_{0}}\right]^{-2\left(1+\rho_{1}\right)}\left[\frac{1-\cos \alpha_{0}}{1-\cos \alpha_{1}}\right]^{-2 \rho_{1}} \\
& =u_{2}\left[\frac{\sin \alpha_{1}}{\sin \alpha_{2}}\right]^{-2\left(1+\rho_{2}\right)}\left[\frac{1-\cos \alpha_{2}}{1-\cos \alpha_{1}}\right]^{-2 \rho_{2}}
\end{aligned}
$$

Hence, it can be found the value for $u_{1}$. Moreover, this equation (49) can be solved for the value of $\alpha_{1}$ such that

$$
\alpha_{1}=2\left[\tan ^{-1}\left[\frac{u_{2}}{u_{0}} \frac{\left(1-\cos \alpha_{2}\right)^{-2 \rho_{2}}\left(\sin \alpha_{0}\right)^{-2\left(1+\rho_{1}\right)}}{\left(1-\cos \alpha_{0}\right)^{-2 \rho_{1}}\left(\sin \alpha_{2}\right)^{-2\left(1+\rho_{2}\right)}}\right]^{\frac{1}{2 \rho_{1}-2 \rho_{2}}}\right]
$$

From equations (43) and (44) the two steps trajectory can be evaluated for vertical range and horizontal span:

$$
\begin{aligned}
& \delta y=\frac{2 u_{1}^{2}}{g_{l}} Y\left(\alpha_{1}, \rho_{1}\right)-\frac{2 u_{0}^{2}}{g_{l}} Y\left(\alpha_{0}, \rho_{1}\right)+\frac{2 u_{2}^{2}}{g_{l}} Y\left(\alpha_{2}, \rho_{2}\right)-\frac{2 u_{1}^{2}}{g_{l}} Y\left(\alpha_{1}, \rho_{2}\right) \\
& \delta x=\frac{2 u_{1}^{2}}{g_{l}} X\left(\alpha_{1}, \rho_{1}\right)-\frac{2 u_{0}^{2}}{g_{l}} X\left(\alpha_{0}, \rho_{1}\right)+\frac{2 u_{2}^{2}}{g_{l}} X\left(\alpha_{2}, \rho_{2}\right)-\frac{2 u_{1}^{2}}{g_{l}} X\left(\alpha_{1}, \rho_{2}\right)
\end{aligned}
$$

The solution is to specify $\alpha_{0}, \alpha_{2}, u_{0}$ and $u_{2}$ and search for reasonable values $N_{1}$ and $N_{2}$ to generate flight path space. An expected trajectory can be chosen from available options. A sample reference trajectory space is shown in Fig. 9. Boundary condition for velocity vector pitch angles were set at $89^{\circ}$ and $0.1^{\circ}$. The initial and final speeds were set at $1688 \mathrm{~m} / \mathrm{sec}$. and $8 \mathrm{~m} / \mathrm{sec}$. The gravity is considered as constant in this analysis where the values for thrust to mass ratios, $N_{1}$ and $N_{2}$ are varied from $0.1 \mathrm{~N} / \mathrm{kg}$ to $10 \mathrm{~N} / \mathrm{kg}$ with $0.25 \mathrm{~N} / \mathrm{kg}$ increments. Fig. 9 shows the impact of varying the thrust acceleration and it appears to have set of curves whereas each curve is created varying $N_{1}$ and $N_{2}$ simultaneously. Three dimensional views are demonstrated in Figs. 10 and 11.

\section{Conclusion}

Precise and safe landing is one of the essential conditions to investigate scientifically interesting area of the lunar surface. Based on the advanced analytical solutions of lunar descent scheme and the proposed algorithm for reference trajectory generation, an accurate representation of lunar descent from orbital condition is demonstrated in this paper, which will make more precision landing. The reference trajectory can be generated in real time, because the state variables were obtained analytically. By making use of such an advanced analytical solution having some reasonable assumptions like including centrifugal acceleration term, computational burden on real-time lunar descent guidance algorithms can be reduced significantly for future landing mission. 


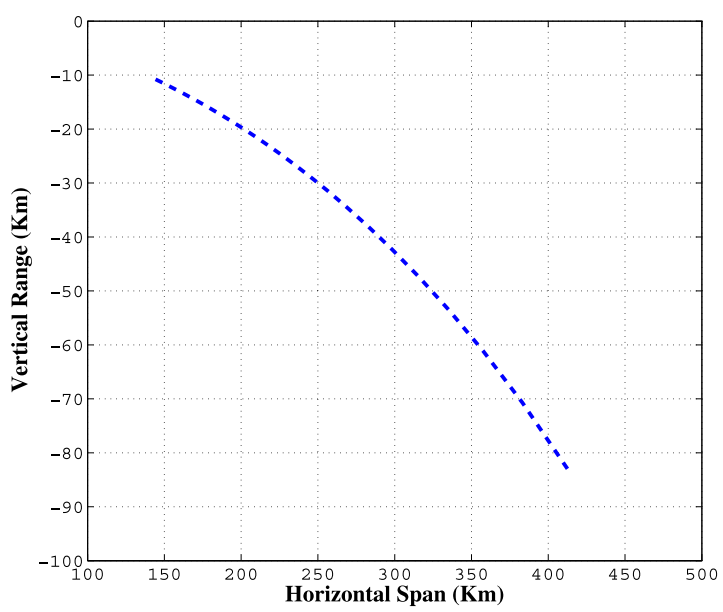

Fig. 9 Sample trajectory space varying $N$

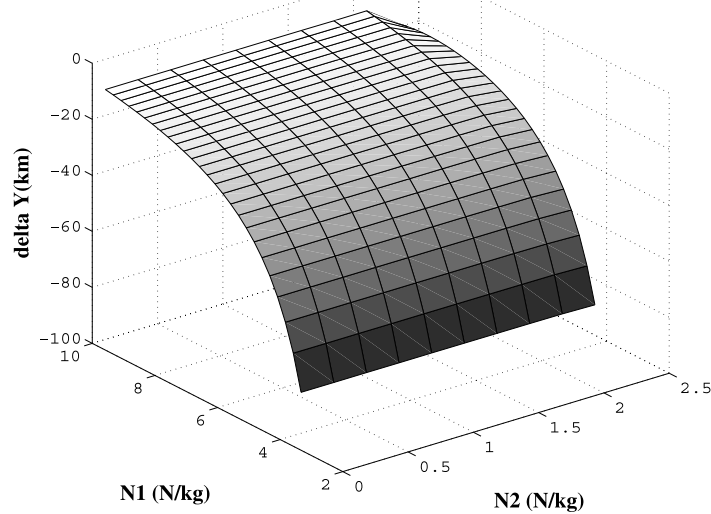

Fig. $103 \mathrm{D}$ view of vertical range sample trajectory space

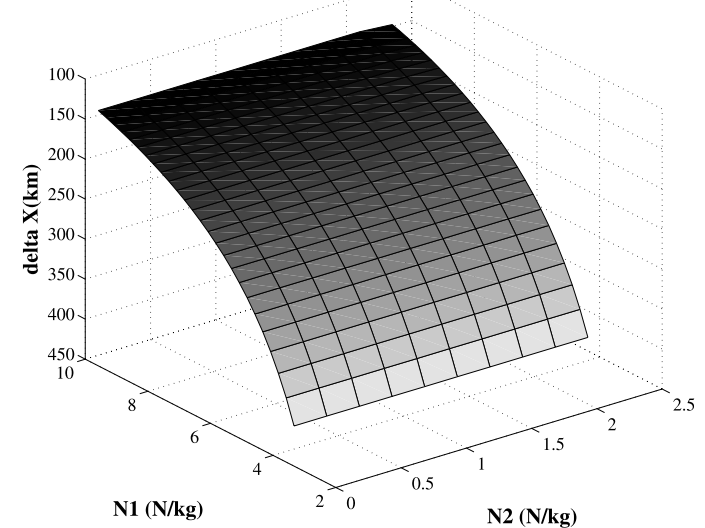

Fig. 11 3D view of horizontal span sample trajectory space 


\section{Acknowledgment}

This work was supported by the Japanese Government's MEXT Scholarship and Global Center of Excellence (GCOE) of the University of Tokyo. It was also supported by the research grant of Japan Aerospace Exploration Agency (JAXA).

\section{References}

( 1 ) Klumpp, A.R., Apollo guidance, navigation, and control: Apollo lunar-descent guidance, Technical report, MIT Charles Stark Draper Laboratory, 1971.

( 2 ) Cheng, R.K., Lunar terminal guidance, In: Lunar Missions and Exploration, edited by C. T. Leondes and R. W. Vance, Univ. of California Engineering and Physical Sciences Extension Series, 308-355, Wiley, New York, 1964.

( 3 ) Cheng, R.K., Meredith, C.M. and Conrad, D.A., Design Considerations for Surveyor Guidance, Journal of Spacecraft and Rockets, 3(11), 1569-1576, 1966.

( 4 ) McInnes, C.R., Direct adaptive control for gravity-turn descent, Journal of Guidance Control and Dynamics, 22(2), 373-375, 1999.

( 5 ) McInnes, C.R., Gravity-Turn Descent from Low Circular Orbit Conditions, Journal of Guidance Control and Dynamics, 26(1), 183-185, 2003.

( 6 ) Ueno, S. and Yamaguchi, Y., Near-Minimum Guidance Law of a Lunar Landing Module, 14th IFAC Symposium on Automatic Control in Aerospace, 377-382, 1988.

( 7 ) Sostaric, R., Lunar descent reference trajectory, Technical report, NASA/JSC, 2006.

( 8 ) Liu X.L., Duan G.R. and Teo K.L., Optimal Soft Landing Control for Moon Lander, Automatica, 44, 1097-1103, 2008.

( 9 ) Bei C. and Zhang W., A Guidance and Control Solution for Small Lunar Probe PreciseLanding Mission, Acta Astronautica, 62, 44-47, 2008.

(10) Scheeres, D.J., Interactions between ground-based and autonomous navigation for precision landing at small solar-system bodies, Telecommunication and data acquisition progress report, 42-132, 1998.

(11) Uchiyama, K., Shimada, Y. and Nakajima, S., Tracking control to near-optimal trajectory for a lunar lander, 23rd International Symposium on Space Technology and Science, 1, 977-982, 2002

(12) Uchiyama, K., Guidance law for lunar lander with input constraint, AIAA Guidance, Navigation and Control Conference and Exhibit, Hilton Head, Soth Carolina, 2007

(13) Pashall, S.C., Brady, T., Cohanim, B.E. and Sostaric, R., A self contained method for safe and precise lunar landing, IEEE Aerospace Conference, 2008.

(14) Mehedi, I.M. and Kubota, T., Advance descent scheme for lunar landing, 18th IFAC Symposium on Automatic Control in Aerospace, Nara, Japan, 2010. 